\title{
Drug eluting stents for the treatment of bare metal in- stent restenosis: long-term outcomes in real world practice.
}

\begin{abstract}
AIMS:

Drug eluting stents (DES) have had a great impact in reducing in-stent restenosis (ISR) in de novo lesions. However, long-term data regarding effectiveness and safety of these stents in treating bare metal stent (BMS) ISR are limited. We report long-term clinical outcomes in a cohort of patients with BMS-ISR treated with DES between April 2002 and December 2003 at our institution.
\end{abstract}

\section{METHODS AND RESULTS:}

Sixty-nine consecutive patients with significant BMS-ISR were treated with DES implantation. Sirolimus DES were used in 43 patients and paclitaxel DES in 26. All patients were followed up to determine the incidence of major adverse cardiac event (MACE) rates (all-cause death, myocardial infarction, or target vessel revascularisation [TVR]), angina class and the need for clinically driven angiography. The mean age of the cohort was $58.6 \pm 10.8$ years; $68 \%$ were male, $33 \%$ were diabetic, $50 \%$ had hypertension, $78 \%$ were on statin therapy and $59 \%$ were current (19\%) or previous (41\%) smokers. The clinical presentation of ISR was with chronic stable angina in 54 patients, 12 had a non-ST elevation acute coronary syndrome and three presented with ST-elevation myocardial infarction. Multivessel stenting was performed in 21 patients and bifurcation stenting in seven patients. Over a mean follow period of 4.9 years, the first event MACE rate was 20\% (17 events in 14 patients - eight deaths of which three were cardiac, two non-fatal myocardial infarctions and seven TVR). Excluding non-cardiac death, the adjusted MACE rate was 14.5\% (12 events in 10 patients). At longterm follow-up, mean Canadian angina class decreased from $2.3 \pm 0.7$ pre-procedure to $1.2 \pm$ $0.4,65 \%$ of patients were angina free and $80 \%$ were free of MACE. No differences in longterm outcomes were observed between patients receiving paclitaxel and sirolimus DES.

\section{CONCLUSIONS:}

The use of DES for the treatment of BMS-ISR is safe and effective over a mean follow-up period of nearly five years. To our knowledge, this represents the longest follow-up data of real world patients treated in a single interventional centre. 